\title{
An Introduction to Soft Matter
}

\author{
Gerhard Gompper and Michael Schick
}

\section{What Is Soft Matter?}

All matter is made of atoms and molecules, and in most common systems, such as water, silicon, or sodium chloride, the size of these building blocks is on a length scale of ångströms. Such atomic structure cannot be discerned by a microscope with a resolution of tens of nanometers, so that the material looks completely homogeneous. The situation is quite different for a suspension of latex particles, or a mixture of oil, water, and surfactant molecules. Even at a resolution of micrometers, the inhomogeneous structure of these materials is still visible. Figure 1 shows a transmission electron microscopy (TEM) image of a colloidal crystal that clearly shows its structure to be visible on the micrometer scale.

What makes these colloidal systems interesting is that their properties can be quite different from those of liquids or crystals of small molecules. For example, materials that consist of extremely large molecules are usually easily deformable. Hence they are usually referred to as "soft matter". The reason for the softness of such macromolecular materials is readily understood. Let us compare the energies that are needed to deform the surfaces of a common molecular crystal, such as sodium chloride, and of a crystal of spherical colloidal particles with a size of $1 \mu \mathrm{m}$, a factor of $10^{4}$ larger than ordinary molecules. The resistance of a crystal with respect to external shear forces is characterized by the shear modulus. For a crystal of linear sizes $L_{x}, L_{y}$, and $L_{z}$, to which a force $F$ is applied at the top and bottom surfaces in the $x$ and $-x$ directions, respectively (see Fig. 2), the shear modulus, $\mu$, is defined by

$$
\frac{F}{L_{x} L_{y}}=\mu \frac{\Delta L_{x}}{L_{z}}
$$

Soft Matter, Vol. 1: Polymer Melts and Mixtures. Edited by G. Gompper and M. Schick Copyright (C) 2006 WILEY-VCH Verlag GmbH \& Co. KGaA, Weinheim ISBN: 3-527-30500-9 


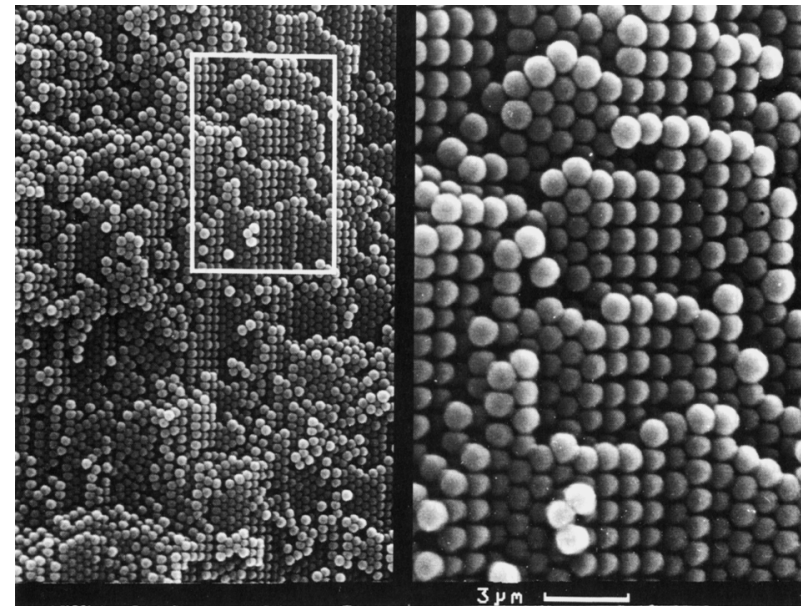

Fig. 1 Transmission electron microscopy (TEM) picture of a colloidal crystal of spherical particles. The figure on the right is an enlargement of the part indicated by the white square in the figure on the left. The ordered arrangement of the colloidal particles is clearly visible.

where $\Delta L_{x}$ is the crystal displacement in the top layer. The shear modulus is an intrinsic property of the material, independent of the system size, and has the dimension energy/(length) ${ }^{3}$. We obtain a rough estimate of the shear modulus of a material by inserting the characteristic length and energy scales of the crystal. For a molecular crystal, the energy scale is that of covalent bonds, $10 \mathrm{eV}$, while for a colloidal crystal the energy scale is not that of some microscopic interaction potential, but rather is on the order of the thermal energy $k_{\mathrm{B}} T$, about $(1 / 40) \mathrm{eV}$ at room temperature. Thus the energy scale is two orders of magnitude smaller in colloidal crystals. The length scale in the colloidal crystal is of the order of its lattice constant, which is four orders of magnitude larger than those of molecular crystals. One therefore estimates that the shear modulus of a colloidal crystal is $10^{-2} /\left(10^{4}\right)^{3}=10^{-14}$ or 14 orders of magnitude smaller than the shear modulus of a molecular crystal. This estimate reproduces quite well experimentally observed values for the shear modulus of colloidal crystals. As the shear modulus is so small, colloidal crystals are extremely soft and can be destroyed by mechanical forces very easily.

The appearance of the thermal energy as the scale of interaction energy is pervasive in soft matter, and reflects the fact that many of the important interactions in these systems are entropic in origin. These effective interactions often arise from microscopic hard-core potentials between the particles themselves, or between the particles and confining walls, or from other constraints. Their net effect is to reduce the allowed configurations of the system 

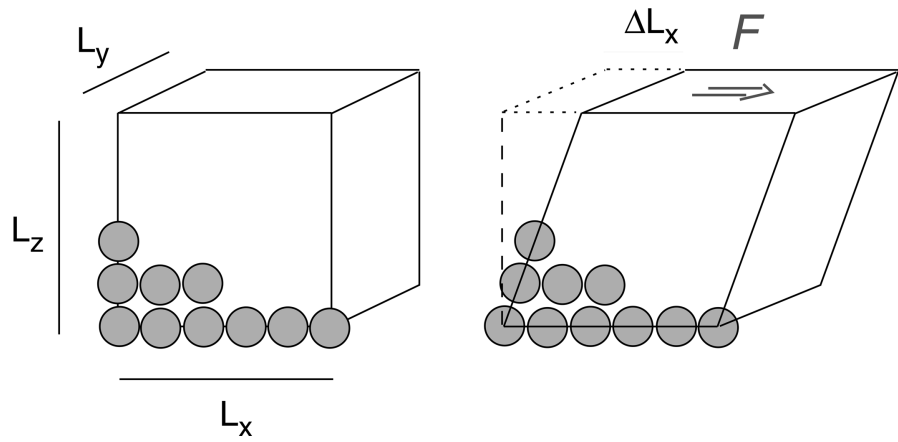

Fig. 2 When a crystal is sheared by a force $F$ applied to its top surface, it is deformed by a distance $\Delta L_{x}$.

and therefore its entropy, and to increase the system's free energy, an increase that can be assigned to an effective interaction. The presence of a factor of $k_{\mathrm{B}} T$ in the magnitude of such interactions belies their entropic origin (Israelachvili 1992).

In addition to colloids, there are many other kinds of very large "molecules". Aggregates of polymers and of smaller amphiphilic molecules are the most common building blocks of soft matter. These blocks will be considered in more detail in the next section.

For reasons similar to those sketched above for colloidal crystals, one finds that membranes consisting of amphiphilic molecules are also easily deformable. Such biological membranes enclose red blood cells, shown in Fig. 3, whose size is on the order of micrometers. Due to the flexibility of its enclosure, a red blood cell can penetrate through openings that are ten times smaller than itself.

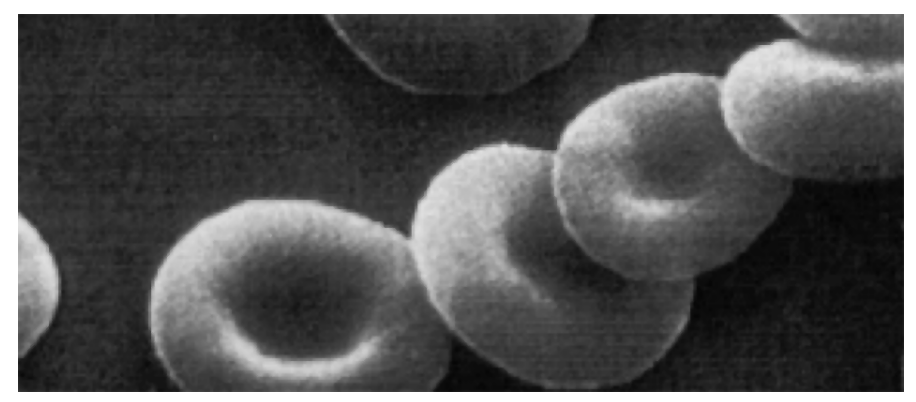

Fig. 3 Red blood cells (erythrocytes) have a characteristic, discocyte shape. 


\section{The Classical Systems}

The "classical" soft matter systems, which have been studied since the early 19th century, are dispersion colloids, systems of small amphiphiles, and polymers. We briefly introduce these systems here.

\section{Dispersion Colloids}

Colloids are rigid particles that have a size in the range of $1 \mathrm{~nm}$ to $10 \mu \mathrm{m}$. They are usually dispersed in a fluid because, in its absence, the colloidal system behaves as a powder. Such solutions are commonly referred to as dispersions or suspensions. The colloidal particles are small enough to exhibit thermal motion, and therefore behave as "large molecules". Their thermal motion is commonly referred to as Brownian motion, named after the Scottish botanist Robert Brown, who observed thermal motion of pollen grains through a microscope in 1827.

Since colloidal particles typically have a different dielectric constant than the embedding fluid, they attract each other by van der Waals interactions (Mahanty and Ninham 1976; Israelachvili 1992), with the result that they are unstable to coagulation. Two methods are commonly employed to stabilize such systems. The first is to charge the colloidal particles electrically with charges of the same sign to produce an electrical repulsion. The second is to coat the surfaces with a polymer layer, which leads to a steric repulsion.

The lower limit on the size of a colloidal particle of approximately $1 \mathrm{~nm}$ assures that the fluid in which the colloidal particles are embedded behaves for the most part as a structureless continuum. Since the typical size of a solvent molecule is $1 \AA$, the colloidal particle can usually be regarded as a macroscopic object on the solvent length scale. Moreover, the relaxation times of the degrees of freedom of the solvent molecules are much smaller than those for the colloidal particles. Dynamics and non-equilibrium phenomena can thus be described by equations of motion in which the degrees of freedom of the solvent molecules are integrated out. For both the structure and the dynamics, a colloidal system of identical colloidal particles can therefore be regarded as an effective one-component system. The solvent now appears only via phenomenological parameters, such as the viscosity, and acts as a thermal motor that drives the motion of the colloidal particles.

Colloidal dispersions in some aspects behave much like ordinary condensed matter. Therefore, they provide interesting model systems from which insight can be gained on fundamental processes that apply to molecular systems as well, such as crystallization, critical phenomena, and glass formation. Indeed, as Dogic and Fraden state in their introduction to Chapter 1 of Volume 2 , they were originally motivated to study virus suspensions because they 


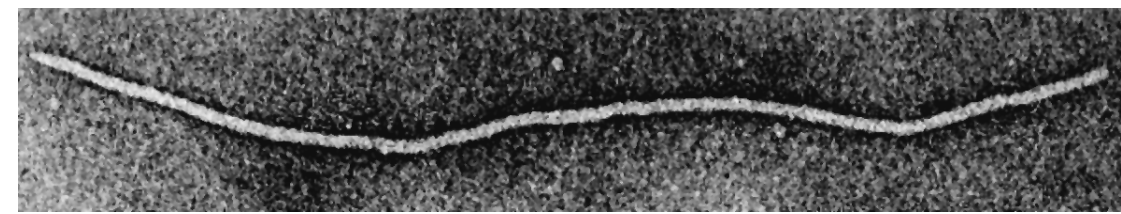

Fig. 4 An $f d$ virus has the shape of a long, semi-flexible rod. It has a diameter of about $6 \mathrm{~nm}$, a length of $880 \mathrm{~nm}$, and persistence length of about $2.2 \mu \mathrm{m}$.

thought that they were model systems of simple fluids. They acknowledge in the very same sentence, however, that their colloidal system, like so many others, has its own specific properties that have no counterpart in molecular systems. There are several reasons why colloids exhibit such specific phenomena. One is that the particles can exist in many different shapes, some of which have been examined extensively: for example, spheres, such as in silica suspensions (Manoharan et al. 2003); rigid rods, such as tobacco mosaic virus and double-stranded DNA fragments; and semi-flexible rods, such as the $f d$ virus shown in Fig. 4. The fascinating phase behavior of this bacteriophage is the subject of Chapter 1 of Volume 2 by Dogic and Fraden. As shown there, these colloids self-assemble into various ordered arrays, a property that is shared by other soft matter systems, such as small amphiphiles, and polymers. It is also one of great technological interest, as the periodicity of the structure can often be tuned by varying the constituent particle. The search for a suitable three-dimensional photonic band-gap material has naturally focussed on the above materials (Vos et al. 1996; Pan et al. 1997; Dinsmore et al. 1998). This property of self-assembly, particularly into ordered arrays, is another recurrent theme in soft matter.

Another reason for the particular behavior of colloids is their very slow dynamics. The microstructural arrangement of colloidal particles typically relaxes in a time range of seconds. This results, for example, in a nonlinear response of colloidal systems, like those of rigid rods, to a shear field, a subject treated authoritatively in Chapter 3 of Volume 2 by Dhont and Briels. For molecular systems, such nonlinear response would occur at unrealistically high shear rates and frequencies. In general, the subject of the hydrodynamic behavior of colloidal fluids is one of significant technological, as well as intrinsic, interest.

Colloids are treated extensively in many texts, such as Russel (1987), Russel et al. (1989), van de Ven (1989), Hunter (1989), Evans and Wennerström (1994), Dhont (1996), and Dhont et al. (2002). 


\section{Self-Assembling Amphiphilic Systems}

Amphiphilic molecules usually consist of a hydrophilic head-group and a hydrophobic tail. The latter typically consists of one or two hydrocarbon chains, which can be totally saturated or partially unsaturated with one or more double bonds. The hydrophilic part consists of either a polar group, whose dipole moment interacts strongly with those of water, or an ionizable group, such as $\mathrm{COOH}$, which dissociates, leaving a residual charge that interacts even more strongly with the solvent dipoles. Such an ionic amphiphile with a tail of a single chain, sodium dodecyl sulfonate, is shown in Fig. 5. An example of a non-ionic, two-chain biological lipid, phosphatidyl choline, is shown in Fig. 6. Note that one chain is saturated, while the other has a sole double bond half-way down the chain. This is a common lipid motif. Molecules such as these have several names: "amphiphilic" from the Greek for "loving both", or "amphipathic", a name favored in the biological community, or "surfactant", a contraction of "surface-active", because these molecules absorb preferentially at interfaces of water with oil or with air and reduce the surface tension.

The basis for the hydrophobic interaction between the tails and water is once again entropic. The tails break up the extensive hydrogen-bonding network of the water, thus reducing its entropy, which can again be viewed as an effective repulsion. The head-groups also disturb this network, but they more

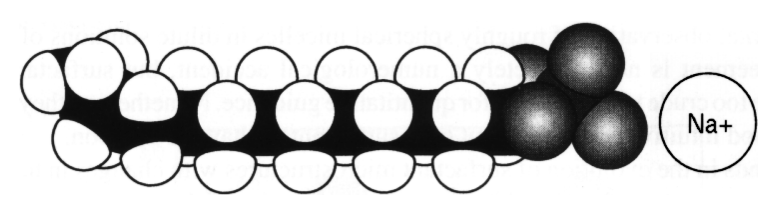

Fig. 5 Sodium dodecyl sulfonate (SDS) is a typical example of an ionic amphiphile. The ionizable head-group (right) is linked to one hydrocarbon chain (left).

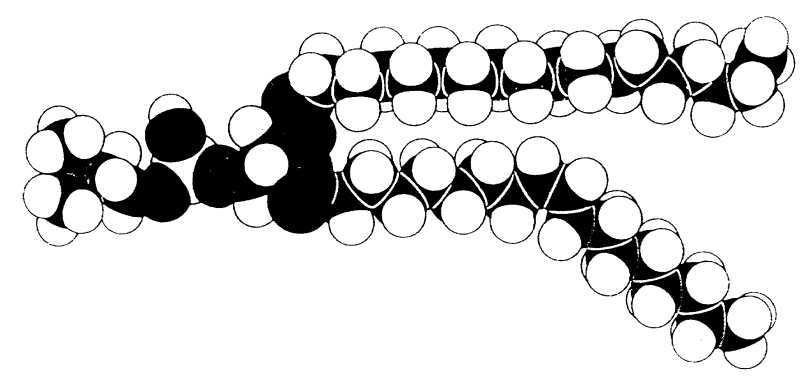

Fig. 6 Phosphatidyl choline (PC) is a typical example of a membrane lipid. The polar head-group (left) is connected to two hydrocarbon tails (right). 

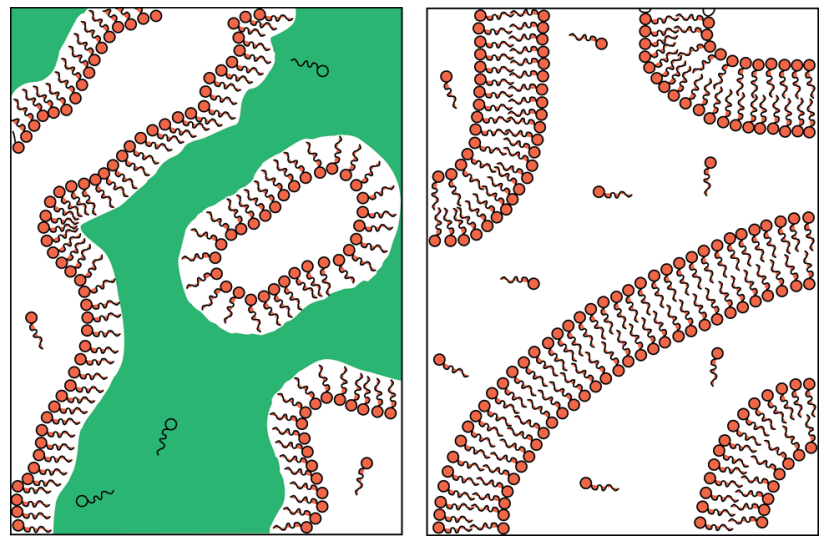

Fig. 7 Amphiphilic molecules self-assemble into complex aggregates due to the hydrophobic effect. In mixtures with oil and water, they form monolayers at the water-oil interface. In mixtures with water, they often form bilayers.

than make up for the entropy reduction by means of their favorable interaction with the water dipoles. Due to these interactions, amphiphiles placed in water will self-assemble and form arrays such that the hydrophilic head-groups are exposed to water while the tails are sequestered from it. The simplest such array is that of a bilayer, shown in Fig. 7, which is a very common structure. Indeed, the basis of all biological membranes is the lipid bilayer. If the amount of amphiphile is increased, the number of bilayers formed is increased, and the system will arrange itself into an ordered lamellar phase. Under various conditions, such as a change in the water content, or a change in the architecture of the amphiphile, several other ordered phases can be formed. Among these are: the cylindrical phase, in which the amphiphiles form single sheets that close into cylinders and are packed in a hexagonal array with the water on the outside and the tails hidden on the inside; a body-centered cubic (bcc) phase, in which the sheets form spheres that pack into a bcc array with water on the outside; and, perhaps most bizarre of all, a "gyroid" phase, in which a bilayer membrane divides space into two identical, sample-spanning, volumes. Because of this, the phase is often denoted "bicontinuous". It is a perfectly good cubic phase (space group $I a \overline{3} d$ ), and was first identified in small amphiphilic systems by Luzzati and Spegt (1967). Later it was observed in systems of block copolymer (Hadjuk et al. 1994). While not uncommonly exhibited by soft materials, it is not a space group ever found in molecular crystals, probably because the structure would consist of atoms in sites of three-fold coordination creating a rather low-density structure not favored by the strong atomic interactions. A disordered and therefore liquid analog of such a bicontinuous phase is observed in these binary (water and amphiphile) 
systems, and has been denoted a "sponge" phase (Cates et al. 1988). When water is removed from these systems, inverted phases may form in which the reduced amount of water is on the inside of the arrays, and the tails are on the outside (Seddon and Templer 1993).

In addition to the ordered phases at relatively high concentration of amphiphile, there is also a fluid phase at low amphiphile concentration in which these molecules form small, usually spherical, aggregates, called micelles. The head-groups face the water and the tails are again sequestered within.

If a third, oil-like, component is added to these systems, it will swell the regions in which the tails reside, as shown in Fig. 7. Thus one finds exactly the same phases as in the binary system but with at least one new possibility. If the concentration of amphiphile is low in a system in which the solvent is oil, the fluid will contain micelles that are inverted, with the heads in and tails out. As one changes the composition of the solvent from mostly water, containing normal micelles with heads out, to mostly oil, containing inverted micelles, the fluid must pass through a region in which the amphiphiles assemble in some intermediate way. In fact, the system can pass through a composition region in which the oil and water are separated by disordered sheets of amphiphiles, again forming a bicontinuous fluid. Such a phase, which still retains much structure, is denoted a microemulsion. In addition to the intrinsic interest of describing a fluid with significant structure, microemulsions have been of technological use, as they can contain in one fluid phase a large

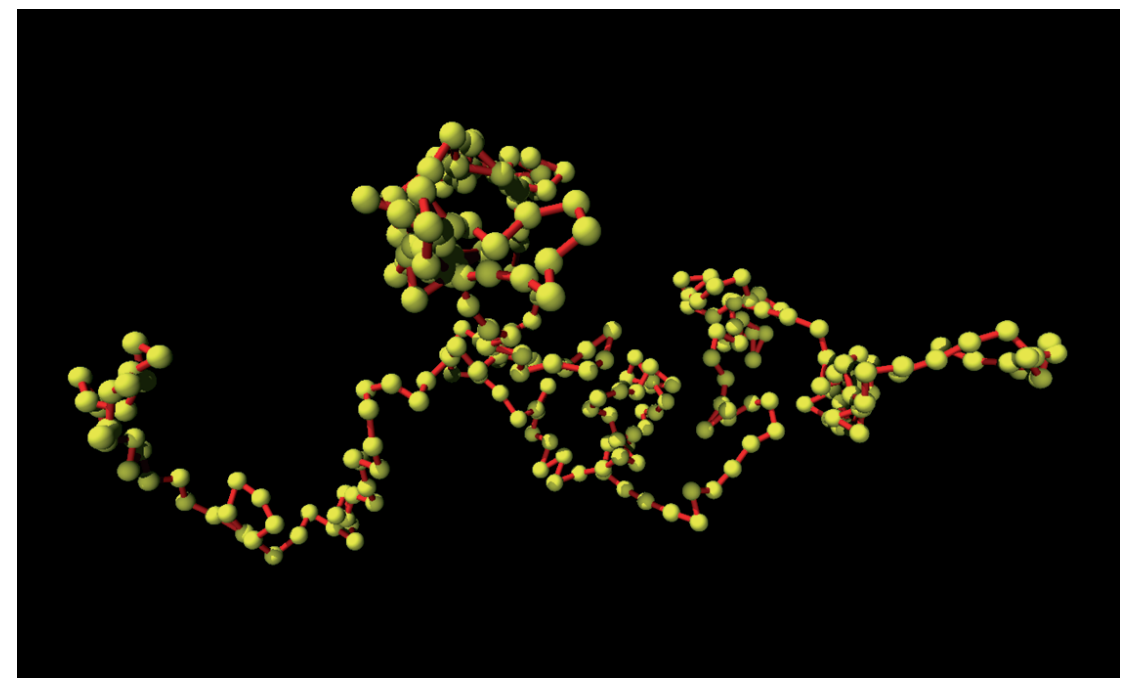

Fig. 8 The typical conformations of long, flexible polymers are random coils. These conformations can be characterized by an end-to-end distance $R_{\mathrm{e}}$, or, equally well, by a radius of gyration $R_{\mathrm{g}}$. 
amount of two components, here oil and water, which would phase-separate in the absence of the amphiphile.

Self-assembling amphiphilic systems are discussed in the texts by Israelachvili (1992), Gelbart et al. (1994), Gompper and Schick (1994), Safran (1994), Jönsson et al. (1998), and Dhont et al. (2002). Texts devoted to the physics of membranes are Lipowsky and Sackmann (1995), Boal (2002), and Nelson et al. (2004).

\section{Polymers}

Polymers are essentially giant molecules, or macromolecules, consisting of an extremely large number of basic units chemically joined together to form one entity. They are ubiquitous. Polyethylene, the practically endless repetition of a simple $\mathrm{H}-\mathrm{C}-\mathrm{H}$ group, forms most of the plastics found in the home, whether in containers, shopping bags, or toys. Polystyrene is the basis for Styrofoam, and also forms the plastic in light switches and the plates surrounding them. Poly(methyl methacrylate) forms the clear plastics Lucite and Plexiglass. Not all polymers, however, are of the technical variety; DNA and RNA are biological polymers consisting of only four distinct monomers, while proteins are also polymers built out of 20 different monomers, the amino acids.

The fact that polymers are ubiquitous should not blind one to the difficulty of accepting the concept of a giant molecule. It is not now, nor was earlier, an obvious one. It was in 1922 that the German organic chemist, Hermann Staudinger, who coined the term "macromolecule", published a theory that rubber was made of a natural polymer, and that it was the entropic properties of such chains that were the source of the elasticity of the material. A long 21 years passed before these insights were recognized by the award of a Nobel Prize in chemistry.

Individual monomers are bound together by covalent bonds, about which rotations are usually possible. Therefore, long polymer chains have a very large number of internal degrees of freedom, which leads to an enormous number of spatial conformations of the chain, and hence a very large entropy. A typical configuration of a flexible polymer is shown in Fig. 8. On distances that are large compared to the size of a monomer, the chemical structure of the building block plays only a minor role, and the properties of the chain are mainly determined by the statistical mechanics of the chain, i.e. essentially by the entropy of a chain. As a consequence, on large distance scales, or for a large number of monomers, $N$, one expects that polymer properties, rather than depending upon the nature of the monomer, will be universal, depending only on the nature of the chain that the monomers form, i.e. whether flexible or semi-flexible, self-intersecting or self-avoiding. The simplest example of 
such a law is the behavior of the chain's radius of gyration, $R_{\mathrm{g}}$, with $N$ :

$$
R_{\mathrm{g}} \sim N^{\nu}
$$

where $\nu$ equals $1 / 2$ in all dimensions for a self-intersecting, completely flexible chain, and is equal to 1,3/4, and 0.5886 in one, two (Nienhuis 1982), and three dimensions (Muthukumar and Nickel 1987) respectively for a self-avoiding chain.

The derivation and elucidation of such scaling laws have played an important role in polymer theory (de Gennes 1979; Doi and Edwards 1986; des Cloizeaux and Jannink 1990), and they are an example of the scaling relations emblematic of critical phenomena. Consequently, the theoretical methods characteristic of that area of study, the use of field theory, the renormalization group, of epsilon expansions, etc., have played a major role. These powerful methods are employed in Chapter 2 of Volume 2 by Eisenriegler to capture the behavior of polymers whose entropy is curtailed by walls and other boundaries.

Because different polymers have different desirable properties, it is natural to try to combine them to create a substance with several such features. There are two common ways to do this. The first is the obvious one of making a simple blend of the two polymers. The problem with this approach is that polymers generally phase-separate, which can be seen as follows. The effective interaction between monomers of different polymers is almost invariably repulsive, i.e. monomers of one kind interact more attractively with their own kind than with a different species of monomer. The number of such interactions is proportional to the total number of monomers, $n N$, where $n$ is the number of chains. However, the entropy is not proportional to the total number of monomers, as it would be were the monomers unlinked; rather, it is only proportional to the number of polymers, $n$. Thus the entropic tendency to mix is much smaller than the energetic propensity to phaseseparate. To counteract this tendency, one plays the same game as in mixtures of oil and water and adds an amphiphile. To solubilize a mixture of A and B homopolymers, one can employ a block copolymer consisting of a block of A monomers chemically joined to a block of B monomers. The diblock will tend to adsorb at the A-B interface and reduce its surface tension. Because the properties of this interface are so important for the blend, it has been the focus of much study. The first technique of choice to be applied to this, as well as most other problems, is that of self-consistent field theory, the subject of Chapter 2 by Matsen, where an entire section devoted to blends can be found. This theory ignores the effects of fluctuations, of course, but their effect can be captured by Monte Carlo simulations, the subject of Chapter 3 by Müller, where a section on blends can also be found. In fact, improvements in both 
models and theories have led to an exciting synergy of these two methods, as detailed in Chapter 3.

A second approach to produce a material with the desirable properties of more than one homopolymer is to utilize block copolymers consisting of several blocks, of which at least two are those of the desired homopolymers. Like other amphiphiles, these block copolymers self-assemble into a variety of ordered one-, two-, and three-dimensional phases, which are of great potential technological use. To pick at random one application: with the use of block copolymer lithography, ordered hexagonal arrays of holes $20 \mathrm{~nm}$ across and $40 \mathrm{~nm}$ apart have been fabricated in a silicon-nitride-coated silicon wafer. The template is made from a polystyrene-polybutadiene diblock that spontaneously self-assembles into a hexagonal phase (Park et al. 1997). It is in the accurate description of such ordered phases, over a wide range of temperatures, that self-consistent field theory has made great strides recently. Again, these are ably reviewed in Chapter 2 by Matsen.

The dynamics of such large polymeric molecules depends, of course, on just how large they are. For long chains, topological constraints absent in normal molecular fluids dominate the dynamics. The constraints are that the polymers cannot break into smaller pieces and cannot cross one another. As a consequence, the polymers become entangled. A particular polymer cannot move normal to its own contour, but only slide along the "tube" formed by the other polymers (de Gennes 1981). Given that the polymer within one tube is part of the boundary of yet another tube makes the problem all the more complex. This difficult but fascinating area of polymer dynamics forms the subject of Chapter 1 by Wischnewski and Richter.

Many fine texts are devoted to polymers. Among them are Flory (1969), de Gennes (1979), Ferry (1980), Doi and Edwards (1986), des Cloizeaux and Jannink (1990), Grosberg and Khokhlov (1997), Hamley (1998), and Dhont et al. (2002).

\section{Why Soft Matter?}

In the past 10-20 years, the research areas of colloids, membranes, microemulsions, and polymers, which were previously largely independent, have been integrated into the single field of soft matter. This is not just a matter of old wine in new bottles; rather, it has been realized that many phenomena in these systems have the same underlying physical mechanisms, so that similar effects do not have to be discovered and understood anew in each subfield. Moreover, combinations of basic elements, such as polymers and colloids, exhibit new properties, which are not found in either system 
separately. From the brief overview of the classical systems above, we can extract several unifying themes.

\section{Model Systems for Atomic Condensed Matter}

Colloidal particles exhibit many of the physical phenomena that are found in ordinary molecular systems. Due to their large size and slow dynamics, however, the experimental study of colloids is usually much simpler than for molecular systems. For example, instead of X-ray or neutron scattering utilized in molecular systems, light scattering can often be employed. The time scales of dynamical phenomena are often in the range of seconds, much longer than the scales of molecular systems.

\section{Tunable Interactions}

In a soft matter system, the interaction between macromolecules arises indirectly from the basic Coulomb and quantum-mechanical interaction of their constituents and those of the surrounding solvent. As the solvent has many degrees of freedom that are subject to thermal fluctuations, the resulting effective interactions are usually temperature-dependent. They can often be controlled and tuned by additives to the solvent.

A good example is the effective interaction potential between colloidal particles, which can be manipulated to vary from a purely long-range repulsion to a very short-range attraction. The tuning of this potential can be accomplished in several ways:

1. Grafting polymers and varying solvent quality. The surface of a colloidal particle can be covered by polymer chains. When the solvent is a good one for the polymers attached to the surface, and the polymer chains are very short in comparison to the size of the core of the particle, the chains do not interpenetrate much, as this would cause a loss of entropy. As a result, the interaction is effectively a hard-core potential: essentially infinite when the inter-particle separation is smaller than the size of the core, and zero otherwise. On the other hand, if the solvent is a poor one for the polymer, in addition to the hard-core repulsion, the interaction has a short-range attractive part because the chains will interdigitate to some extent in order to escape the effects of the solvent. The quality of the solvent, which affects the interaction between the polymers anchored to the colloidal particles, can be varied simply by changing the temperature. One effectively changes the depth of the attractive well between particles.

2. Employing the depletion interaction. Another possibility is to add long, free, polymers to the solvent. Because they are excluded from the region between particles when those particles come close to one another, there results an 
imbalance in the osmotic pressure, leading to an effective attraction. This attraction, which is completely entropic in origin, is denoted the depletion interaction. The range of the attraction is set by the size of the excluded polymers, while the interaction strength is set by their number concentration, as would be expected from the simple van't Hoff relation for osmotic pressure. This polymer-induced interaction is discussed in detail by Eisenriegler in Chapter 2 of Volume 2.

3. Charging the colloids. If the surface of the colloidal particles is charged, one can change the range of the repulsion by adding or removing salt from the solvent, and thereby changing the Debye screening length.

4. Manipulating the dielectric properties. The refractive index of the solvent can also be varied, which changes the strength of the van der Waals attractions between the cores of the colloidal particles.

Such mechanisms for tuning effective interactions are not restricted to colloids. In a very similar way, the interactions between membranes can be varied by anchoring polymers to them, or by adding polymers to the solution, or by adding salt if the membranes are charged.

\section{Universality}

Many phenomena of soft matter are independent of the particular chemical structure of the molecular building blocks. An example discussed above is the relation between the size of a polymer, as measured by its average end-to-end distance, and the physical length of the polymer, which is determined by the number of its monomers. Similar scaling relations appear in the description of the behavior of membranes and colloids.

In addition to this universality, there is also a universality of phase behavior among the different classes of materials. We have already discussed the fact that colloids, amphiphiles, and block copolymers all self-assemble into ordered phases. This is a consequence of effective interactions that are repulsive at short distances and attractive at long distances. Consequently, there is an intermediate scale that is favored by the system, one which sets the scale for the ordered phases. Although the sequence of ordered phases is most commonly manifest in a multitude of soft matter systems, they are also displayed in the small quantum systems of two-dimensional electron gases where the mobile charges form ordered states (Fogler et al. 1996) right up to the astronomical systems of neutron stars in which protons and electrons order (Pethick and Potekhin 1998)! (Obviously they attract one another at large distances. They also repel one another at short distances if they do not have sufficient energy to form a neutron at the top of the Fermi sea.) 
Another example of different systems with the same behavior is that of rodlike micelles, which can be so long that they exceed their persistence length many times over and then behave as "living polymers". What is meant is that in many respects they behave as polymers, so that many of their properties can be predicted readily. However, their dynamics are not the same because long micelles can fission into shorter ones and vice versa; i.e. they do not have the same topological constraints as ordinary polymers.

\section{Soft Matter Composites}

More and more, systems are being investigated that contain several components of the classical materials, such as mixtures of colloids and polymers, polymers attached to membranes, colloids in microemulsions, and emulsion droplets in a nematic solvent. In order to understand the behavior of these systems, knowledge must be drawn from the various subfields of soft matter. A very nice example of the complexity that can arise in soft matter systems is the mixture of rod-like colloids and polymers (Dogic and Fraden 2001) discussed by Dogic and Fraden in Chapter 1 of Volume 2. In the regime of very small colloid concentrations, the colloid particles are found to form a thermally fluctuating membrane. The interaction that keeps the colloids together is the depletion interaction, discussed briefly above. The colloidal rods in this experiment are the $f d$ viruses shown in Fig. 4 . Thus, these are not stiff rods, but semi-flexible objects, and therefore display polymer-like behavior. Note that the thickness of this membrane is now given by the length of the $f d$ virus, i.e. about $1 \mu \mathrm{m}$; this is about three orders of magnitude larger than the thickness of usual lipid bilayer membranes. Finally, the $f d$ virus itself is a biological polymer, which is enclosed by a self-assembled layer of amphiphilic proteins. Thus, this system behaves as a colloidal, an amphiphilic, and a polymeric system on several different scales.

\section{Loomings}

It is clear that there are at least two very strong currents in contemporary soft matter research. The first is that of nanotechnology and new materials; the second is that of biology. Both of these exploit the self-assembling nature of systems, and the malleability and variability of composites. In this, the first of a series of volumes devoted to pedagogical reviews of new and exciting topics in soft matter, we have tried to assemble some of the basics required for our journey of exploration. We can only surmise where this newly launched vessel will carry us, but we can surely guarantee that the voyage itself will be an exciting one! 


\section{References}

Boal, D. H., 2002, Mechanics of the Cell. Cambridge University Press, Cambridge.

Cates, M. E., Roux, D., Andelman, D., Milner, S. T., and Safran, S. A., 1988, Europhys. Lett. 5, 733.

de Gennes, P.-G., 1979, Scaling Concepts in Polymer Physics. Cornell University Press, Ithaca, NJ.

de Gennes, P.-G., 1981, Macromolecules 14, 1637.

des Cloizeaux, J. and Jannink, G., 1990, Polymers in Solution. Clarendon Press, Oxford.

Dhont, J. K. G., 1996, An Introduction to Dynamics of Colloids. Elsevier, Amsterdam.

Dhont, J. K. G., Gompper, G., and Richter, D. (eds.), 2002, Soft Matter Complex Materials on Mesoscopic Scales, Vol. 10 of Matter and Materials. Forschungszentrum Jülich, Jülich.

Dinsmore, A. D., Crocker, J. C., and Yodh, A. G., 1998, Curr. Opin. Coll. Interface Sci. 3, 5.

Dogic, Z. and Fraden, S., 2001, Phil. Trans. R. Soc. Lond. A 359, 997.

Doi, M. and Edwards, S. F., 1986, The Theory of Polymer Dynamics. Clarendon Press, Oxford.

Evans, D. F. and Wennerström, H., 1994, The Colloidal Domain, Where Physics, Chemistry, Biology and Technology Meet. VCH Publishers, New York.

Ferry, J. D., 1980, Viscoelastic Properties of Polymers. John Wiley \& Sons, New York.

Flory, P. J., 1969, Statistical Mechanics of Chain Molecules. Interscience, New York.

Fogler, M. M., Koulakov, A. A., and Shklovskii, B. I., 1996, Phys. Rev. B 54, 1853.

Gelbart, W. M., Ben-Shaul, A., and Roux, D. (eds.), 1994, Micelles, Membranes, Microemulsions, and Monolayers. Springer-Verlag, Berlin.

Gompper, G. and Schick, M., 1994, in Phase Transitions and Critical Phenomena, eds. C. Domb and J. Lebowitz, Vol. 16, pp. 1-176. Academic Press, London.

Grosberg, A. Y. and Khokhlov, A. R., 1997, Statistical Physics of Macromolecules. Springer, New York.

Hadjuk, D. A., Harper, P. E., Gruner, S. M., Honeker, C. C., Kim, G., Thomas, E. L., and Fetters, L. J., 1994, Macromolecules 27, 4063.

Hamley, I. W., 1998, The Physics of Block Copolymers. Oxford University Press, Oxford.

Hunter, R. J., 1989, Foundations of Colloid Science. Clarendon Press, Oxford. 
Israelachvili, J. N., 1992, Intermolecular and Surface Forces. Academic Press, London.

Jönsson, B., Lindman, B., Holmberg, K., and Kronberg, B., 1998, Surfactants and Polymers in Aqueous Solution. John Wiley, Chichester.

Lipowsky, R. and Sackmann, E. (eds.), 1995, Structure and Dynamics of Membranes - From Cells to Vesicles, Vol. 1 of Handbook of Biological Physics. Elsevier, Amsterdam.

Luzzati, V. and Spegt, P. A., 1967, Nature 215, 701.

Mahanty, J. and Ninham, B. W., 1976, Dispersion Forces. Academic Press, London.

Manoharan, V. N., Elsesser, M. T., and Pine, D. J., 2003, Science 301, 483.

Muthukumar, M. and Nickel, B. G., 1987, J. Chem. Phys. 86, 460.

Nelson, D., Piran, T., and Weinberg, S. (eds.), 2004, Statistical Mechanics of Membranes and Surfaces, 2nd edn. World Scientific, Singapore.

Nienhuis, B., 1982, Phys. Rev. Lett. 49, 1062.

Pan, G., Kesavamoorthy, R., and Asher, S. A., 1997, Phys. Rev. Lett. 78, 3860.

Park, M., Harrison, C., Chaikin, P. M., Register, R. A., and Adamson, D. H., 1997, Science 276, 1401.

Pethick, C. J. and Potekhin, A. Y., 1998, Phys. Lett. B 427, 7.

Russel, W. B., 1987, The Dynamics of Colloidal Systems. University of Wisconsin Press, London.

Russel, W. B., Saville, D. A., and Schowalter, W. R., 1989, Colloidal Dispersions. Cambridge University Press, Cambridge.

Safran, S. A., 1994, Statistical Thermodynamics of Surfaces, Interfaces, and Membranes. Addison-Wesley, Reading, MA.

Seddon, J. M. and Templer, R. H., 1993, Phil. Trans. R. Soc. Lond. A 334, 377.

van de Ven, T. G. M., 1989, Colloidal Hydrodynamics. Academic Press, London.

Vos, W. L., Sprik, R., van Blaaderen, A., Imhof, A., Lagendijk, A., and Wegdam, G. H., 1996, Phys. Rev. B 53, 16231. 